confirming the unfitness to drive of those whose conduct has resulted in threatened or actual accidents, or in indicating what is amiss in them and what may be amenable to correction. They will also prove useful in selecting the best applicants for driving public vehicles and vehicles owned by commercial or industrial companies.

If stringent selection is impossible, and if proper mechanical safeguards have been introduced, training is the next most important prevention against accidents. Clearly, the pedestrian and the bicyclist cannot be selected; it is therefore essential that they, above all others, should receive instruction how to avoid accidents from motor traffic. There is one truth which the experienced industrial psychologist never tires of asserting, that no one should be permitted to pick up his methods as best he can-not even the road user! Systematic training is essential for all road users, including the car driver. Yet at present no systematic training is required even from the latter. A still wider problem of psychological importance relates to the most effective forms of propaganda and of appeal to the public for the improvement of road behaviour. The methods devised by the industrial psychologist in market research would prove of great value here. These methods have hitherto been employed to save the waste of time, effort and expense which is so often incurred when the psychologically untrained industrialist places an advertisement or a new design or package of goods in the market in the mere belief that it will be commercially successful. Market research consists in ascertaining systematically and directly what the general public desires and prefers, and in ascertaining the differences in desire and preference due to sex, age, social level, etc. Experience in such market research has enabled the industrial psychologist to avoid various pitfalls which await the inexpert, untrained person. If his methods were applied to the discovery from all angles of the most attractive, least resented forms of propaganda and appeal to the general public in regard to the avoidance of dangers on the road, this would prove to be yet another psychological approach to the problem of road accidents.

Thus there are many ways in which the special abilities and training of the applied psychologist can be utilised-in the design of traffic and car signals and car controls, in the nature and multiplicity of traffic regulations, in the illumination of roads and vehicles, in incentives, propaganda and instruction of road users, in inquiries into accidents and defective road conduct, and in the selection and rejection of drivers.

\title{
Progress in the Gas Industry
}

PEOPLE are so accustomed to having gas on tap, requiring only the aid of a match to give them heat or light, that they are prone to forget the gas industry is a progressive one, continually improving on the production side, in quality of product and in the efficiency and adaptability of the apparatus used to burn it. It is a proud boast of the industry that gas excels all other public services in reliability.

In this Jubilee year it is appropriate to summarise the developments throughout the industry during a quarter of a century: this has been happily done in the summer number of West's Gas Journal, which contains articles by several leading experts in the various sections of gas engineering. A brief epitome of the developments should be of interest to a wider public, since coal and its carbonisation form one of the major problems of Great Britain. The use of raw coal in the domestic grate and many other appliances, including the factory boiler and furnace, has been condemned for years, and a prior treatment advocated to recover the volatile constituents leaving the smokeless fuelcoke-for burning. The old customs die but slowly though there now appears to be an acceleration in the disuse of raw coal, judging particularly from the statistics of railway traffic.

Gas is made by carbonising coal in horizontal or vertical retorts or in large chambers. In 1910 vertical retorts were newly introduced; they have since made great strides. The methods of charging the coal and discharging the coke have been greatly improved, as also the manner and economy of heating the retort settings. As a consequence, the number of gaseous therms per ton of coal carbonised has considerably increased.

Since the Gas Regulation Act of 1920, gas has been sold on a declared calorific value and charged for by the therm instead of by volume, though it is this which is actually measured by the meters. The London undertakings have tended to keep a higher calorific value (of 500 B.T.U.) than the provinces, in part owing to the necessity of getting a higher duty out of their distribution mains. It is desirable to emphasise that the great gas companies send out a gas of constant heating value, at a constant pressure, of a constant density and low in sulphur, so that it burns in suitable apparatus 
without any regulation. Coke at one time was regarded as a by-product and its quality varied accordingly ; to-day it is standardised as carefully as is gas and distributed graded as to size, ash content and burning capacity, so that it requires a minimum of supervision when burnt in closed stoves. A great amount of scientific work has been applied to realise these high standards of quality of gas and coke, and the achievement is no mean one. The penetration of the chemist into the gas industry largely coincides with the Jubilee period : he is being regarded more and more as one of the key men in the industry and there are not wanting those who regard its future as largely on the chemical side.

The gas engineer continues to develop improvements in detail and to organise gas matters on a larger scale, including carbonising, purification and distribution. Condensing practice has changed in favour of the horizontal tube form of welded steel construction, electric precipitation removes the last traces of tar. Centrifugal and static type washers are in use, and it has become customary to remove naphthalene and benzole by washing. Unfortunately, the ammonia recovery has received a setback owing to the fall in price due to the competition of the synthetic product. Purifying is almost exclusively done with oxide of iron, which enables the sulphur to be recovered in a marketable form; in some cases the sulphur is almost completely removed by a catalytic process, though generally this problem is still left for the future. For storage purposes, the waterless holder now competes with the old-fashioned gasometer: in Great Britain it is still in the experimental stage in regard particularly to economy of maintenance and the extra supervision required, though both on the Continent and in America it has become widely adopted.

High pressure distribution of gas has brought new problems, largely solved by the use of welded steel tubes. The introduction of spun cast iron pipes has increased their length and given a more homogeneous material than ordinary cast iron.

For a long time the output of the industry increased each year. Latterly, there has come a great change in the domestic habits of the people, and gas also has to face intense competition from other sources of light and heat. In consequence, there is an awakening to salesmanship with the provision of show-rooms. In particular, a great stimulus has been given to the improvement of gas-cookers and fires which, since 1910, have been bettered in all respects almost beyond recognition. The same applies to water heating and to the use of gas for industrial heating, a field in which there is great development taking place. Lastly, gas holds its own for street lighting, as an evening in the streets round Whitehall or in South London will testify. Here the physicist has been called into co-operation so that the maximum light can be directed along the length of the road.

This brief sketch indicates the virility of what is now an old industry, largely due to the more scientific outlook which it is showing towards its problems. There is a tendency to concentrate the industry in larger units, either by regional absorp. tion or amalgamation of the smaller companies by the larger, or by the purchase of isolated companies by holding groups. The former plan obviously makes for greater efficiency and benefit to the consumer; the latter move is regarded with some unrest by many.

\title{
Chemotherapy of Malaria*
}

\author{
By Colonel S. P. James, F.R.S.
}

$\mathrm{W}^{1}$ ITH the distressing effects of the malaria epidemic and famine in Ceylon still fresh in our minds, the subject of the chemotherapy of malaria may very appropriately be discussed at the present time. The area affected was not so large as Yorkshire, but the cost of relief measures was about $£ 350,000$, and during the six months from November until April, $14 \frac{1}{2}$ tons of quinine, costing $£ 50,700$, was used. About $£ 20,000$ was also spent on the new antimalarial remedy called atebrin. The epidemic reminded the world once

\footnotetext{
* Opening of a discussion in Sections B (Chemistry) and E (Geography of the British Association at Norwich on September 9, 1935.
}

again that no royal road or short cut to the prevention of malaria has yet been found. During recent years similar reminders have come from British India, Malaya, East and South Africa, Nigeria, the Sudan, Mauritius, Trinidad, Barbados and other British territories overseas. A few years ago an epidemic in British India prostrated the whole population of an area twice as big as England. The ordinary business of large cities was interrupted, no labour could be obtained, transport was disorganised, and even sellers of food ceased to carry on their trade. In one group of towns and villages the deaths during the month 